1 Grant R, Condon B, Hart I, Teasdale GM. Changes in intracranial CSF volume after lumbar puncture and their relationship to post-LP headache. J Neurol Neurosurg Psychiatry 1991;54:440-2.

2 Kuntz KM, Kokmen E, Stevens JC, Miller P, Offord KP, Ho MM. Post lumbar puncture headaches: experience in 501 consecutive procedures. Neurology 1992:42:1884-7.

3 Strupp M, Brandt T, Muller A. Incidence of post-lumbar puncture syndrome reduced by reinserting the stylet: a randomised prospective study of 600 patients. J Neurol 1998;245:589-92.

4 Greene HM. Lumbar puncture and the prevention of post puncture headache. JAMA 1926;86:391-2.

5 Evans RW. Complications of lumbar puncture. Neurol Clin North Am 1998:16:83-105.

6 Carson D, Serpell M. Choosing the best needle for diagnostic lumbar puncture. Neurology 1996;47:33-7.
7 Muller B, Adelt K, Reichmann H, Toyka K. Atraumatic needle reduces the incidence of post-lumbar puncture syndrome. J Neurol 1994;241:376-80. 8 Kleyweg RP, Hertzberger LI, Carbaat PAT. Significant reduction in post-lumbar puncture headache using an atraumatic needle. A double-blind, controlled clinical trial. Cephalalgia 1998;18:635-7.

9 Braune HJ, Huffman G. A prospective double-blind clinical trial, comparing the sharp Quincke needle (22G) with an "atraumatic" needle (22G) in the induction of post-lumbar puncture. Acta Neurol Scand 1992;86:50-4.

10 Flaatten H, Krakenes J, Vedeler C. Post-dural puncture related complications after diagnostic lumbar puncture, myelography and spinal anaesthesia. Acta Neurol Scand 1998;98:445-51.

11 Lybecker H, Moller JT, May O, Nielsen HK. Incidence and prediction of postdural puncture headache. A prospective study of 1021 spinal anesthesias. Anesth Analg 1990;70:389-94.

(Accepted 17 July 2000)

\title{
Reliability of Snellen charts for testing visual acuity for driving: prospective study and postal questionnaire
}

\author{
Zanna Currie, Archana Bhan, Irene Pepper
}

\section{Editorial \\ by Westlake \\ Ophthalmology Department, \\ Royal Hallamshire \\ Hospital, Sheffield \\ S10 2JF \\ Zanna Currie \\ specialist registrar \\ Archana Bhan \\ senior house officer \\ Irene Pepper \\ specialist registrar \\ Correspondence to: \\ Z Currie \\ zanna@zcurrie. \\ freeserve.co.uk}

BMJ 2000;321:990-2

\begin{abstract}
Objectives To assess the ability of patients with binocular $6 / 9$ or $6 / 12$ vision on the Snellen chart (Snellen acuity) to read a number plate at $20.5 \mathrm{~m}$ (the required standard for driving) and to determine how health professionals advise such patients about driving.

Design Prospective study of patients and postal questionnaire to healthcare professionals. Subjects 50 patients with $6 / 9$ vision and 50 with $6 / 12$
\end{abstract} vision and 100 general practitioners, 100 optometrists or opticians, and 100 ophthalmologists.

Setting Ophthalmology outpatient clinics in Sheffield. Main outcome measures Ability to read a number plate at $20.5 \mathrm{~m}$ and health professionals' advice about driving on the basis of visual acuity.

Results $26 \%$ of patients with $6 / 9$ vision failed the number plate test, and $34 \%$ with $6 / 12$ vision passed it. Of the general practitioners advising patients with $6 / 9$ vision, $76 \%$ said the patients could drive, $13 \%$ said they should not drive, and $11 \%$ were unsure. Of the general practitioners advising patients with $6 / 12$ vision, $21 \%$ said the patients could drive, $54 \%$ said they should not drive, and 25\% were unsure. The level of acuity at which optometrists, opticians, and ophthalmologists would advise drivers against driving ranged from $6 / 9^{-2}$ (ability to read all except two letters on the $6 / 9$ line of the Snellen chart) to less than $6 / 18$.

Conclusions Snellen acuity is a poor predictor of an individual's ability to meet the required visual standard for driving. Patients with 6/9 vision or less should be warned that they may fail to meet this standard, but those with $6 / 12$ vision should not be assumed to be below the standard.

\section{Introduction}

Drivers must ensure that their vision is good enough to drive, but people commonly seek the advice of healthcare professionals on this matter. The legal standard required for driving a private car or motorbike (group 1 entitlement) is to be able to read a number plate at $20.5 \mathrm{~m}$. Guidelines issued by the Driver and Vehicle
Licensing Authority suggest that this corresponds to between $6 / 9$ and $6 / 12$ vision on the Snellen chart (Snellen acuity), ${ }^{1}$ and the guidelines of the Royal College of Ophthalmologists equate this to about $6 / 10$ vision. However, $6 / 10$ does not exist on standard Snellen charts, and professionals might be led to assume that all those with $6 / 9$ vision meet the required standard and that those with $6 / 12$ do not.

We aimed to determine what percentage of patients with $6 / 9$ and of those with $6 / 12$ vision could pass a number plate test. We then assessed by postal questionnaire the advice given by healthcare professionals.

\section{Participants and methods}

\section{Participants}

Fifty patients with binocular $6 / 9$ vision and 50 with $6 / 12$ vision were recruited prospectively from ophthalmology clinics. Their ability to read a number plate was tested out of doors at a distance of $20.5 \mathrm{~m}$. Snellen acuity was measured uniocularly and binocularly. We included patients if they could see the full line on the Snellen chart binocularly and no more than two letters on the line below. The patients were given two attempts at reading a standard front number plate (registration number F758 EKY). Some numbers and letter-number combinations are harder to see than others. ${ }^{2}$ The number plate we chose represented a moderately difficult combination. All tests took place between 9 am and 3 pm during good weather. The patients wore their usual glasses, but the accuracy of the refraction of the lenses was not tested. We did not assess visual fields or driving status.

\section{Questionnaires}

Questionnaires were sent by post to 100 ophthalmologists throughout Great Britain and 100 optometrists or opticians in South Yorkshire (boxes). A modified questionnaire went to 100 general practitioners in South Yorkshire.

\section{Results}

\section{Number plate test}

The $6 / 9$ and $6 / 12$ vision groups each comprised 21 men and 29 women, with a mean age of 78 (range 43 


\section{Questions to general practitioners}

A patient comes to you asking if his or her vision is good enough to drive. You measure the patient's visual acuity as $6 / 9$ on a Snellen chart and ascertain that there are no other reasons to preclude driving (that is, reduction in visual field or diplopia). Would you tell the patient he or she could drive? (Answers: "yes," "no," or "don't know.")

If "no" or "don't know" would you refer to any one else or are there any other tests that you would do or information that you would seek? (Please elaborate.) If you measure the patient's visual acuity as $6 / 12$ on a Snellen chart and ascertain that there are no other reasons to preclude driving, would you tell the patient he or she could drive?

If "no" or "don't know" would you refer to any one else or are there any other tests that you would do or information that you would seek? (Please elaborate.)

to 95 ) and 73 (range 18 to 94 ) respectively. Two patients with binocular $6 / 9$ vision had best uniocular acuities of $6 / 12$. In the group with $6 / 12$ binocular vision, five had uniocular acuities of $6 / 9$ but managed only $6 / 12$ binocularly and two had uniocular acuities of $6 / 18$ but achieved the higher standard binocularly. Thirteen patients $(26 \%, 95 \%$ confidence interval $14 \%$ to $38 \%$ ) with binocular $6 / 9$ vision could not read the number plate, including two whose best uniocular acuity was only $6 / 12$ and 11 whose vision in the second eye ranged from $6 / 9$ (five patients) to counting fingers.

Seventeen patients $(34 \%, 21 \%$ to $47 \%)$ in the $6 / 12$ group could read the number plate, including four of the five with a uniocular acuity of $6 / 9$. The acuity in the second eye of the 13 others ranged from 6/12 (six patients) to counting fingers

\section{Questionnaires}

The response rate was $63 \%$ for general practitioners, $60 \%$ for optometrists and opticians, and $72 \%$ for ophthalmologists. Based on a Snellen acuity of $6 / 9,76 \%$ of the general practitioners would tell patients that they could drive, $13 \%$ would advise against it, and $11 \%$ were unsure. Based on a Snellen acuity of $6 / 12,21 \%$ of the general practitioners would tell patients that they could drive, $54 \%$ would advise against it, and $25 \%$ were unsure. Of the 18 who were unsure about either question, $94 \%$ would refer the patient to either their optician, the guidelines of the Driver and Vehicle Licensing Authority, or the Highway Code.

The table summarises the responses from optometrists and ophthalmologists regarding the level of acuity at which patients are advised that they should not drive. Thirteen (13 of 60;22\%) optometrists advised "borderline" cases to self assess, and nine oph-

\section{Questions to optometrists and ophthalmologists}

When clients or patients ask you whether or not they can drive, providing that they have no diplopia or reduction in visual fields, at what level of acuity do you tell them that they should not drive, and how would you measure it?

Are there any other tests that you would do or information that you would seek? thalmologists (9 of $72 ; 13 \%)$ quoted no Snellen acuity but advised self assessment. Of the ophthalmologists mentioning the required standard, four quoted an incorrect distance.

\section{Discussion}

The number plate viewed at $20.5 \mathrm{~m}$ subtends a visual angle of 13.4 minutes of arc, geometrically corresponding to $6 / 15$ on the Snellen chart. The guidelines linking Snellen acuity and driving come from a statistical model. This was derived by Drasdo and Haggerty on the basis of 28 candidates failing the vision test for driving, whose binocular acuity was correlated with the distance at which they could read a number plate. ${ }^{3}$ The model estimated that $6 / 9^{-2}$ (the ability to read all except two letters on the $6 / 9$ line of the Snellen chart) would produce the same failure rate as the number plate test, and some authorities extrapolated this to $6 / 10$. The probability of passing the test with an acuity of $6 / 7.5$ or $6 / 18$ was predicted as $99 \%$ and $6 \%$ respectively. Our study agrees broadly with this but highlights the difficulties of predicting an individual's chance of success. As our patients had eye disease, they may have performed worse than a normal cohort, where fewer patients might fail with $6 / 9$ vision but more patients might pass with $6 / 12$ vision.

The questionnaire responses showed that drivers receive a spectrum of advice. Establishing a level of Snellen acuity at which to advise patients against driving could represent the upper limit, below which caution is advised, or the lower limit, below which all are advised to stop. Thus none of the responses is necessarily incorrect if accompanied by advice on the legal requirements. However, for patients with binocular Snellen acuity of $6 / 9$ or less inquiring about their eligibility to drive, the only correct response is "I don't know," unless the health professional tests their ability to read a number plate outside.

Surveys have shown that a low proportion of the public know the visual requirements for driving, and other studies have shown that a major proportion of drivers would not pass the number plate test. ${ }^{4-6}$ The advantage of the number plate test is its amenability, but the practicalities of measuring the distance and self assessing can be off-putting for some. If those testing vision could provide a way for people to self assess-for example, by mounting a number plate at the correct

Cut-off point of visual acuity at which opticians, optometrists, and ophthalmologists would advise patients against driving. Values are numbers (percentages) of healthcare professionals

\begin{tabular}{lcc} 
Snellen acuity & Optometrists and opticians $\mathbf{(} \mathbf{n} \mathbf{6 0})$ & Ophthalmologists $(\mathbf{n}=\mathbf{7 2})$ \\
\hline $6 / 9^{-2}$ & $15(25)$ & $6(8)$ \\
\hline $6 / 10$ & $1(2)$ & $4(6)$ \\
\hline $6 / 12$ & $15(25)$ & $19(26)$ \\
\hline$<6 / 12$ & $11(18)$ & $20(28)$ \\
\hline $6 / 15$ & $3(5)$ & $2(3)$ \\
\hline $6 / 18$ & $4(7)$ & $10(14)$ \\
\hline$<6 / 18$ & $4(7)$ & $1(1)$ \\
\hline Number plate chart & $4(7)$ & $9(13)$ \\
\hline Number plate outside† & $3(5)$ & 9
\end{tabular}

*Indoor test with simulated number plate similarly mounted to Snellen chart and subtending same angle as number plate at $20.5 \mathrm{~m}$.

tIncludes all health professionals who would only offer this advice to patients or who would actively test patients in this way. 
What is already known on this topic

It is estimated that a Snellen acuity of $6 / 9^{-2}$ produces the same failure rate as the number plate test at $20.5 \mathrm{~m}$, leading to guidelines that a visual acuity between $6 / 9$ and $6 / 12$ is required for driving

\section{What this study adds}

A proportion of patients with binocular $6 / 9$ vision failed to read a number plate at $20.5 \mathrm{~m}$, and a major proportion with binocular $6 / 12$ vision could read it

Snellen visual acuity is a poor indicator of an individual's chance of meeting the legal standard for driving, and all people with a visual acuity of $6 / 9$ or less should be encouraged to self assess their vision

Patients receive a spectrum of advice from healthcare professionals about driving on the basis of a visual acuity of $6 / 9$ or $6 / 12$

distance outside the practice-this would provide a useful public service.

\section{Conclusions}

Snellen acuity is a poor indicator of an individual's chance of passing a number plate test, and it cannot be assumed that a driver with a visual acuity of $6 / 9$ will meet the standard for driving. All drivers with 6/9 vision or less must be encouraged to self assess. Conversely, it should not be assumed that a driver with a visual acuity of $6 / 12$ is below the standard for driving, particularly as loss of the right to drive has such far reaching social implications. ${ }^{78}$ Advice that vision required for driving lies somewhere between $6 / 9$ and $6 / 12$ acuity could cause confusion if these points are not stressed.

AB's current address is Queens Medical Centre, Nottingham NG7 2UH.

Contributors: ZC contributed to the design of the study and questionnaire, distribution of the questionnaire, patient testing, analysis of the results, and writing the paper; she will act as guarantor. $\mathrm{AB}$ contributed to the distribution of the questionnaire, patient testing, and writing the paper. IP contributed to the analysis of the results, patient testing, and writing the paper.

Funding: None.

Competing interests: None declared.

1 Driver and Vehicle Licensing Authority. At a glance guide to the current medical standards of fitness to drive. Swansea: Drivers Medical Unit, DVLA, 1998:25-6.

2 Marsden AM, Packer AL. The visibility of vehicle number plates. Trans Illuminating Eng Soc (London) 1966;31:2.

3 Drasdo N, Haggerty CM. A comparison of the British number plate and Snellen vision tests for car drivers. Ophthalmol Physiol Opt 1981;1:39-54.

4 Taylor SP. Accuracy of recall of the legal number plate testing distance by UK drivers. Ophthalmol Physiol Opt 1997;17:473-7.

5 Department of Transport. Consumer trends survey Aug, 1998

6 McCaghrey GE. AOP vision screening 1985/86. Optom Today 1987;27:178-81.

7 Shipp MD, Penchanski R. Vision testing and the elderly driver: is there a problem meriting policy change? J Am Optom Assoc 1995;66:343-51.

8 Charman WN. Visual standards for driving. Ophthalmol Physiol Opt 1985;5:211-20.

(Accepted 24 July 2000)

\section{Unjustified exclusion of elderly people from studies submitted to research ethics committee for approval: descriptive study}

Antony Bayer, Win Tadd

Correspondence to: A Bayer

bayer@cf.ac.uk

continued over

BMJ 2000;321:992-3

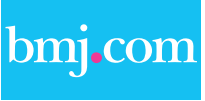

This article is part of the BMJ's randomised controlled trial of open peer review. Documentation relating to the editorial decision making process is available on the BMJ's website
Ageism in clinical practice ${ }^{1}$ and published research ${ }^{2}$ is well recognised. We were interested in whether research protocols submitted to the local research ethics committee contained unjustified upper age limits and how the committee dealt with this.

\section{Methods and results}

We reviewed all studies submitted to Bro Taf local research ethics committee in the first seven months of 1999 to determine whether any upper age limits were justified and whether the committee had commented on such age restrictions. We then made a judgment on the appropriateness of the upper age limit.

Of 225 studies whose protocols were reviewed, 65 were on topics or conditions that automatically excluded elderly people. Five studies specifically concerned elderly people and had a lower age limit but no upper limit. Of the remaining $155,90(58 \%)$ had an upper age limit, which ranged from 45 years (in a smoking cessation intervention) to 100 years (in a study of an open access mental health service), with a median of 70 years (interquartile range 65 to 75 years). In five studies an upper age limit was reasonable (participants were required to have no important disease, or prolonged follow up was planned).

In 85 studies the age restriction was inappropriate and unnecessary, but ethical review had failed to highlight this issue. Justification was offered by researchers in only one study. Age limits often conflicted with the aims of the study-for example, in a study of "subjects randomly selected to reflect each life stage," another "of consecutive patients attending for bone scan," and another that claimed "no special groups to be excluded." In some studies exclusion of older patients was likely to result in an atypical clinical populationfor example, an investigation of "exclusion of vulnerable people from services," studies of type II diabetes, glaucoma, or non-steroidal anti-inflammatory drugs that were restricted to people aged under 65 , and a study comparing incontinence aids that was limited to those aged under 70 . 